$(p=0.001) ; 29 \%(p=0.001) ; 10 \%(p=0.06) ; 27 \%(p=0.009)$, respectively. Although only $26 \%$ of the patients have had an articular US, in $65 \%$ it led to a change in treatment. Positive answers to some questions, which were perhaps too complex, showed a discrete increase, less than $10 \%$, conceivably due to the surprisingly high background of positive answers.

Conclusions: As expected, the US image method was familiar to most but not its applicability in articular diseases. After the lecture there was an increase in understanding of the positive impact of US in rheumatic diseases, implying that short and focused lectures are a useful tool in educational programs for patients.

Disclosure of Interest: None declared

DOI: 10.1136/annrheumdis-2017-eular.1426

\section{SAT0736-HPR "LIKE A BAG OF LIQUORICE ALLSORTS - EVERYBODY'S GOT DIFFERENT FLAVOURS": A QUALITATIVE FOCUS GROUP STUDY TO EXPLORE SYMPTOMS OF FATIGUE, SLEEP DISTURBANCES AND PAIN IN PRIMARY SJÖGREN'S SYNDROME PATIENTS AND TO DEVELOP A FUTURE MODEL OF CARE}

K.L. Hackett ${ }^{1}, 2$, V. Deary ${ }^{2,3}$, K. Deane ${ }^{4}$, J.L. Newton ${ }^{1,2}$, W.-F. Ng $^{1,2}$, T. Rapley ${ }^{1}$ ${ }^{1}$ Newcastle University; ${ }^{2}$ Newcastle upon Tyne NHS Foundation Trust; ${ }^{3}$ Northumbria University, Newcastle upon Tyne; ${ }^{4}$ University of East Anglia, Norwich, United Kingdom

Background: Fatigue, sleep disturbances and pain, are symptoms of primary Sjögren's syndrome (PSS). However, current clinical interventions predominantly focus on treating patients' dryness symptoms.

Objectives: To explore the experience of fatigue, sleep disturbances and discomfort in people with PSS, to investigate the impact of these symptoms on patients' daily lives and to develop an intervention strategy to address them.

Methods: Qualitative focus groups with open-ended questions allowed participants to explore ideas together and focus on issues they perceived as being important. PSS patients $(n=10)$ and spouses $(n=3)$ took part in three focus groups divided into six sessions which were facilitated by two clinician researchers using a topic guide. Discussion topics included; the symptoms, strategies used by patients used to manage their symptoms, possible future intervention components proposed from the literature and possible modes of delivering future interventions. The meetings were audio-recorded, transcribed verbatim and analysed using thematic analysis ${ }^{1}$.

Results: Patient participants all experienced these symptoms. Symptom severity varied within individuals and flares occurred unpredictably. Fatigue, sleep disturbances and discomfort, all affected patients' lives and those around them and sometimes felt overwhelming. Discomfort symptoms included oral and ocular dryness, tingling, nausea and difficulties tolerating light and noise. The invisible nature of these features meant patients often struggled to meet others' expectations, which affected their mood and resulted in social withdrawal. Fatigue was a major barrier to engaging in work, productivity and leisure activities. Sleep disturbances further compounded the fatigue. Patients employed a range of strategies to self-manage their symptoms to varying degrees of success.

Participants expressed a need for tailored support from health care professionals which included information provision, access to peer support and professional support to apply symptom management information. A three stepped model of care was proposed. The model includes different modes of delivering intervention content, including written information, education groups, peer support, digital self-management and one-to-one therapy. Intervention intensity increased with each step in the model.

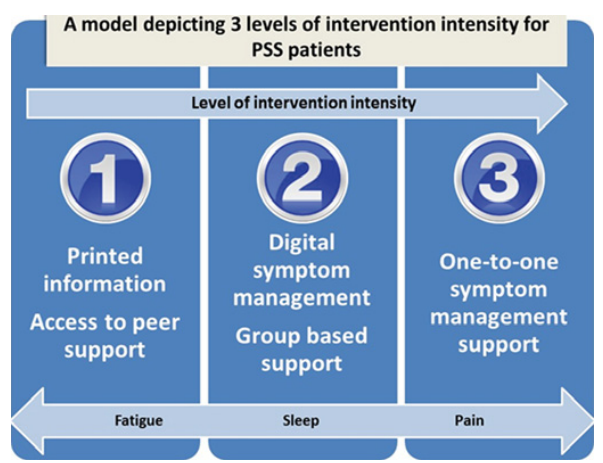

Conclusions: Symptoms of fatigue, sleep disturbances and discomfort all impact on PSS patients' daily lives and individualised interventions are needed to support self-management. Care needs to be tailored as different patients require variable levels of support. A stepped model of future symptom management delivery is proposed.

References:

[1] Braun V and Clarke V (2006) Using thematic analysis in psychology. Qualitative

Research in Psychology, 3(4), 77-101.

Acknowledgements: This project was funded by Arthritis Research UK (grant 20169) and the United Kingdom Occupational Therapy Research Foundation.
Disclosure of Interest: None declared

DOI: 10.1136/annrheumdis-2017-eular.2823

\section{SAT0737-HPR FACILITATORS AND BARRIERS FOR PARTICIPATION IN PHYSICAL ACTIVITIES IN JUVENILE IDIOPATHIC ARTHRITIS PATIENTS AND HEALTHY CONTROLS}

K. Risum ${ }^{1}$, A.M. Selvaag ${ }^{1}$, Ø. Molberg ${ }^{1}$, H. Dagfinrud ${ }^{2}$, H. Sanner ${ }^{1} .{ }^{1}$ Os/o University Hospital; ${ }^{2}$ Diakonhjemmet Hospital, Oslo, Norway

Background: Knowledge is sparse regarding facilitators and barriers for participation in physical activity (PA) in patients with juvenile idiopathic arthritis (JIA) and whether they differ from controls. Furthermore, knowledge about preferences for leisure time physical activities and participation in physical education (PE) in school in JIA patients is limited.

Objectives: To explore participation in PA and PE in JIA patients, and to explore facilitators and barriers for PA participation in JIA and matched controls.

Methods: The study cohort included $60 \mathrm{JIA}$ patients (50 girls, 10 boys) and 60 controls individually matched for age and sex randomly selected from the Norwegian Population Registry. Of the JIA patients, 30 had persistent oligoarthritis and 30 had polyarticular disease (extended oligoarthritis and polyarticular RF $+/$-). The patients were aged 10-16 years and recruited consecutively at Oslo University Hospital in 2015. Participation in PA and facilitators and barriers for PA participation, were explored with structured interviews. The interview guide was developed for this study based on literature review and clinical experience. Differences between the study groups were analyzed using the McNemar test.

Results: Participation in physical activities was not significantly different between JIA patients and controls (Table 1). The most commonly practiced organized physical activities in both groups were dancing and soccer, and the most commonly practiced unorganized physical activities were jogging, training at fitness center and strength exercising at home. Participation in PE is shown in Table 1. Fun was the most reported facilitator for participation in PA both in patients and controls, $40(67 \%)$ vs $45(75 \%)$, respectively, $\mathrm{p}=0.32$. Becoming fit was an often reported facilitator in patients and controls, $12(20 \%)$ vs $21(35 \%)$ respectively, $p=0.07$. Being with friends was a facilitator more often reported by controls than patients, $13(22 \%)$ in controls vs $1(2 \%)$ patient $(p<0.001)$. Less pain was a facilitator in 4 patients, but not in any controls $(p=0.06) .26(43 \%)$ patients and $19(32 \%)$ controls reported barriers for participating in PA $(p=0.46)$. More controls reported time as a PA barrier, $11(18 \%)$ vs $3(5 \%)(p=0.02)$, while more patients reported pain as a barrier; $18(30 \%)$ vs $8(13 \%)(p=0.03)$. Disease activity was a barrier in 4 (7\%) patients.

Table 1. Participation in physical activity (PA) and physical education (PE)

\begin{tabular}{lccc}
\hline & JIA $(n=60)$ & Controls $(\mathrm{n}=60)$ & p-value \\
\hline Participation in PA (organized/unorganized) & $51(85)$ & $56(93)$ & 0.14 \\
Participation in organized PA & $38(63)$ & $47(78)$ & 0.11 \\
Participation in unorganized PA & $41(68)$ & $42(70)$ & 1.00 \\
Participation in PE & $42(70)$ & $59(98)$ & $<0.001$ \\
Always & $16(27)$ & $0(0)$ & \\
Always (sometimes with modification) & $2(3)$ & $1(2)$ & \\
Sometimes & &
\end{tabular}

Numbers are $\mathrm{n}(\%)$

Conclusions: The majority of JIA patients and controls participated in organized or unorganized PA. Fun was the most reported facilitator in patients, followed by becoming fit and having less pain. Pain was the dominant PA barrier in patients, while time was the most frequently reported barrier in controls. Some patients experienced disease activity as a barrier. Nearly all the patients (97\%) participated regularly in $\mathrm{PE}$, but $1 / 4$ needed some modifications. These results are reassuring, and highlight the importance of enjoyment for participation in PA Disclosure of Interest: None declared

DOI: 10.1136/annrheumdis-2017-eular.6063

\section{SAT0738-HPR EXPERIENCES OF TELE-HEALTH FOLLOW-UP IN PATIENTS WITH RHEUMATOID ARTHRITIS: A QUALITATIVE INTERVIEW STUDY}

L.R. Knudsen ${ }^{1}$, A. de Thurah ${ }^{1,2}$, K. Lomborg ${ }^{2,3}$. ${ }^{1}$ Department of Rheumatology, Aarhus University Hospital; ${ }^{2}$ Department of Clinical Medicine, Aarhus University ${ }^{3}$ The Research Programme in Patient Involvement, Aarhus University Hospital, Aarhus, Denmark

Background: Recently, the effectiveness of monitoring disease activity in rheumatoid arthritis (RA) through patient reported outcome (PRO) based telehealth follow-up was tested in a randomized controlled trail, TeRA (1). The TeRA study evaluated the effectiveness of the tele-health follow-up but did not study how patients grasp this new form of disease control. Many studies on tele-medicine focus on the over-all patient satisfaction with tele-medicine follow-up, but gives limited insight into what drives patient satisfaction (2).

Objectives: To explore the experiences of a PRO based tele-health follow-up from the perspective of patients with RA with a special attention on experiences of taking a more active role and assuming more responsibility in the disease control. Methods: The qualitative research strategy was Interpretive Description (3). From October 2015 to January 2016 we conducted individual semi-structured interviews 